\title{
Hypnosis and mindfulness meditation: two third generation behavioral and cognitive therapies
}

Keywords: psychology, commitment therapy, negative cognitions, emotions, hypnosis

Abbrevations: DBT, dialectical behavior therapy; ACT, acceptance and commitment therapy; MBCT, mindfulness-based cognitive therapy; EMDR, eye-movement and reprocessing: WSGC, waterloo-stanford group scale of hypnotic susceptibility, form C

\section{Conceptual paper}

Volume 70, number 7, American Psychologist, a journal of the American Psychological Association, devoted an entire issue to mindfulness based therapies in psychology (American Psychologist, October 2015 70(7) 581-658). Murdock ${ }^{1}$ noted that mindfulness based approaches are the third wave of behavioral and cognitive therapies. ${ }^{1}$ In addition, she stated that Dialectical Behavior Therapy (DBT), Acceptance and Commitment Therapy (ACT), and MindfulnessBased Cognitive Therapy (MBCT) are the most popular of the third wave therapies. This paper will briefly discuss hypnosis and mindfulness meditation as third wave behavioral therapies. Finally, paper also presents effect size data.

Mindfulness Approaches are the third generation of behavioral approaches, ${ }^{2}$ and hypnosis can easily be placed within this generation. The first generation of behavior therapies was stimulus control, contingency management, exposure therapy, and modeling therapies. Stimulus control consists of prompting and setting events that are environmental stimuli or conditions that elicit behavior. ${ }^{3}$ Contingency management involves reinforcement/punishment, token economy, contingency contracts, and behavioral parent training. Exposure therapies are brief/graduated exposure, prolonged/intense exposure. Modeling therapies are vicarious extinction, and skills training.

The second generation consists of cognitive-behavioral therapies: cognitive restructuring and cognitive-behavioral therapies: coping skills. Cognitive restructuring can be further broken down into thought stopping, rational emotive behavior therapy, and cognitive therapy. Coping skills consist of self-instruction training, problemsolving therapy, stress inoculation training, and cognitive-behavioral couples' therapy.

The third generation consists of acceptance and mindfulness-based therapies, and these can be further broken down into acceptance and commitment therapy, dialectical behavior therapy, mindfulness-based cognitive therapy, and ACT.

Holzman, et al. $^{4}$ indicated that postmodern approaches in psychology evolved after the cognitive-behavioral revolution in psychology. ${ }^{4}$ Postmodern psychology is a philosophy that questions if ultimate truth can be obtained; therefore, many approaches or methodologies are used to approximate truth. Postmodern psychology is not a unidimensional construct or theory. Finally, some examples of postmodern approaches are positive psychology, feminist psychotherapy, and multicultural counseling.

\author{
Volume I Issue 4 - 2017 \\ Marty Sapp \\ Department of Educational Psychology, University of Wisconsin- \\ Milwaukee, USA
}

Correspondence: Marty Sapp, Professor, Department of Educational Psychology, University of Wisconsin-Milwaukee, USA, Emailsapp@uwm.edu

Received: November 08, 2017| Published: December 13, 2017

Sapp $^{5}$ stated that hypnosis was a postmodern approach, but it is also part of the mindfulness-based meditation approaches. Actually, acceptance and commitment therapy, dialectical behavior therapy, eyemovement and reprocessing (EMDR), mindfulness-based cognitive therapy, and hypnosis are part of the third generation of behavior therapies. ${ }^{5} \mathrm{ACT}$ as a mindfulness-based meditational approach involves accepting feeling, thought, and behaviors without trying to change them. DBT was developed by Marsha Linehan ${ }^{6}$ to treat the features of borderline personality disorder such as suicidal attempts, self-harming behaviors such as self-cutting. Linehan's approach has the acceptance of feelings and behaviors like ACT. ${ }^{6}$ As previously stated, DBT is a third generation form of cognitive-behavioral therapy. Dialectics are defined as the synthesis of opposing opposites, and It was developed by Dr. Marsha Linehan at the University of Washington, ${ }^{6}$ and she has developed techniques for dealing with more riskier behaviors like suicide, self-mutilation, substance abuse, borderline personality disorder (BPD), and other disorders. Leahy ${ }^{7}$ pointed out that DBT places more of an emphasis on the behavioral dimension of cognitive-behavioral theory. For example, a greater emphasis is placed on teaching clients behavioral skills for regulating negative cognitions, behaviors, and emotions. Finally, DBT has been extended to a variety of psychological disorders.

Eberth, et al. ${ }^{8}$ reported an $\mathrm{r}$ effect size of .27 for mindfulness meditation. In contrast, Sapp ${ }^{5}$ reported an effect size for $r$ of .68 for hypnosis. What is not known is if mindfulness meditation can influence hypnosis measures, or if hypnosis can influence mindfulness measures. Gloede ${ }^{9}$ randomly assigned college participants to a prerecorded 15-minute mindful meditation transcript and another group to a 15-minute hypnosis transcript. After each group listened to the pre-recorded transcript, they were measured on group hypnosis using the Waterloo-Stanford Group Scale of Hypnotic Susceptibility, Form C (WSGC). The purpose of this study was to determine if these two transcripts differed on their impact on group hypnosis, and to find the $\mathrm{d}$ effect size. In a preliminary statistical analysis, Gloede did not a statistically significance difference between hypnosis and mindfulness meditation, and the d effect size was close to zero. ${ }^{9}$ Additional research is needed in this area, and studies are needed that combine hypnosis and mindfulness meditation versus hypnosis alone. Finally, hypnosis needs testing within a mindfulness meditation context. ${ }^{10}$ 


\section{Acknowledgements}

None.

\section{Conflicts of interest}

The author declared that there are no conflicts of interest.

\section{References}

1. Murdock NL. Theories of counseling and psychotherapy: A case approach. 3rd ed. Staten Island, New York, USA: Pearson Higher; 2012.

2. Hayes SC. Acceptance and commitment therapy, relational frame theory, the third wave of behavioral and cognitive therapies. Behav Ther. 2004;47(6):869-885.

3. Spiegler MD. Contemporary behavior therapy. USA: Nelson Education; 2015.

4. Holzman L, Morss JR. Postmodern psychologies, societal practice and political life. London, UK: Routledge; 2000.
5. Sapp M. Hypnosis and postmodernism: Multicultural Applications. Sleep Hypn. 2016;18(1):19-15.

6. Linehan MM. Cognitive-behavioral treatment of borderline personality disorder. New York, USA: Guildford Press; 1993.

7. Leahy RL. Overcoming resistance in cognitive therapy. New York, USA Guildford; 2003.

8. Eberth J, Sedlmeier P. The effects of mindfulness meditation: A metaanalysis. Mindfulness. 2012;3(3):174-189.

9. Gloede M. The power of suggestibility: Hypnosis and mindfulness meditation. USA: Study conducted at the University of WisconsinMilwaukee; 2017

10. Otani A. Hypnosis and mindfulness: the Twain finally meet. Am J Clin Hypn. 2016;58:383-398. 